ARTICLE

Received 28 Aug 2013 | Accepted 26 Nov 2013 | Published 10 Jan 2014 DOI: 10.1038/ncomms4019

\title{
A fully genetically encoded protein architecture for optical control of peptide ligand concentration
}

Daniel Schmidt ${ }^{1}$ Paul W. Tillberg ${ }^{2, \star}$, Fei Chen $^{1, \star} \&$ Edward S. Boyden ${ }^{1}$

Ion channels are among the most important proteins in biology, regulating the activity of excitable cells and changing in diseases. Ideally it would be possible to actuate endogenous ion channels, in a temporally precise and reversible manner, and without requiring chemical cofactors. Here we present a modular protein architecture for fully genetically encoded, lightmodulated control of ligands that modulate ion channels of a targeted cell. Our reagent, which we call a lumitoxin, combines a photoswitch and an ion channel-blocking peptide toxin. Illumination causes the photoswitch to unfold, lowering the toxin's local concentration near the cell surface, and enabling the ion channel to function. We explore lumitoxin modularity by showing operation with peptide toxins that target different voltage-dependent $\mathrm{K}^{+}$channels. The lumitoxin architecture may represent a new kind of modular protein-engineering strategy for designing light-activated proteins, and thus may enable development of novel tools for modulating cellular physiology.

\footnotetext{
${ }^{1}$ Departments of Brain and Cognitive Sciences and Biological Engineering, MIT Media Lab and McGovern Institute, MIT, Cambridge, Massachusetts 02139, USA. ${ }^{2}$ Department of Electrical Engineering and Computer Science, MIT, Cambridge, Massachusetts 02139, USA. * These authors contributed equally to this work. Correspondence and requests for materials should be addressed to E.S.B. (esb@media.mit.edu).
} 
on channels govern cellular signaling and computation, in neurons and neural compartments as well as other excitable cell classes, and are significant drug targets for a variety of disorders $^{1,2}$. Ideally, one could genetically target ion channels for perturbation to assess their causal contribution to complex systems. Earlier studies have approached this problem by several kinds of innovation. For example, one line of inquiry has resulted in genetically encoded membrane-targeted peptide toxins that can be expressed in cell types of interest. Peptide toxins comprise a broad class of genetically encoded ion channel modulators from venomous animals that are capable of recognizing targets from every major ion channel family, with extraordinary specificity ${ }^{3-8}$. These reagents function without requiring exogenously supplied chemicals, and are inducible and reversible over timescales of hours to days ${ }^{9-12}$, and have been shown to function in mammalian brain in vivo. A second line of inquiry has utilized light-responsive chemical modulators that are anchored to specific sites engineered into ion channels (for example, SPARK, LiGluR and HyLighter) ${ }^{13-19}$, and thus are capable of fast induction and reversal with light. They incorporate exogenously supplied engineered chemicals, which bind to engineered sites on targeted ion channels. The first technology is fully genetically encoded but not light-modulatable; the second technology is rapidly light-modulatable but not genetically encoded.

Here we devise a novel protein architecture capable of modulating endogenous ion channels, but which is both fully genetically encoded, and actuatable by light. We accordingly here adapt three different technology building blocks in a novel protein architecture to yield a fully genetically encoded reagent that can actuate endogenous voltage-gated $\mathrm{K}^{+}(\mathrm{Kv})$ channels with light. We create a fusion protein containing a peptide toxin as the ion channel ligand and a light-oxygen-voltage (LOV) domain photoswitch tethered to the cell membrane. We hypothesize that upon illumination, conformational changes in the LOV domain lower the local concentration of the peptide toxin near the cell surface, and thus enables the ion channel target's functionality in response to light. We develop a quantitative theory for the function of these 'lumitoxins' and characterize the biophysical properties of an $\alpha$-dendrotoxinbearing lumitoxin modulating $\mathrm{Kv}$ channels expressed in mammalian cells. We create a library of lumitoxins made of mutated peptide toxin variants to illustrate the tunability of our system. Finally, we establish modularity of the lumitoxin protein architecture by creating several lumitoxins containing genetically encoded $\mathrm{K}^{+}$channel blockers, which upon illumination will disinhibit different subclasses of $\mathrm{Kv}$ channels. Our modular, simple architecture for light-controlled protein ligand concentration may provide a platform for rapidly making new libraries of novel light-actuatable protein ligands that are genetically targetable.

\section{Results}

A theory for light-actuated tethered protein ligands. Owing to the great structural and functional knowledge about potassium channels $^{20,21}$, we focused on $\mathrm{Kv}$ channels for this study. One exemplar implementation of the lumitoxin architecture is to express a fusion protein that contains a $\mathrm{Kv}$ channel-specific peptide toxin (for example, $\alpha$-Dendrotoxin, $\alpha$ DTX), and a photoreceptor domain ${ }^{22,23}$ (for example, LOV2-J $\alpha$ ), targeted to the secretion pathway and anchored to the extracellular side of the cell membrane (Fig. 1a). To acquire some intuition about potential mechanisms of operation of such reagents, we implemented a simplified tethered-particle motion model of this hypothetical fusion protein and simulated its diffusive behaviour to gain insight into the characteristic parameters. Our simulation predicts that the number of peptide collisions with the cell surface is decreased as the tether length is increased, the computational analogue to the unfolding of the $\mathrm{J} \alpha$ helix. Specifically, by inspecting the probability distribution in the $x-z$ plane (that is, looking at the lumitoxin from the side as in Fig. 1a) we get an appreciation of the changes in the mean peptide localization. Shown are the simulation results for a six-membered and a 27-membered membrane linker (Fig. 1b, upper left and lower left panels, respectively). The distribution difference demonstrates that after unfolding of the $J \alpha$ helix, it is more likely to find the peptide $6 \mathrm{~nm}$ away from the membrane plane; the local concentration close to the peptide toxin's binding site on a membrane-bound ion channel would accordingly be greatly reduced (Fig. 1b, right panel).

The effect of this apparent dilution can be understood if we consider an equilibrium system in which a lumitoxin can adopt two different states, Lit (LL) or Dark (LD) (Fig. 1c). Here the litstate of the membrane-tethered peptide toxin has a lower local concentration near the cell surface, resulting in a decreased apparent association rate constant $\left(\alpha^{\star}\right)$ for binding to the channel (Ch) and then blocking it ( $\mathrm{Ch} \times \mathrm{LL}$ or $\mathrm{Ch} \times \mathrm{LD})$. As approximations, we assume that the lumitoxin and channel are expressed to similar levels, and further assume that the dynamics of light-dependent conformational changes in the LOV-J $\alpha$ photoreceptor are independent of the peptide toxin/channel binding equilibrium.

We found that when using published values for the binding of $\alpha$-DTX to $\mathrm{Kv}^{24,25}$ and the light-dependent transitions of an isolated LOV2-J $\alpha$ domain from Avena sativa ${ }^{26,27}$ (see Methods for details), the system equilibrates with most ion channels (>90\%) being occupied by a peptide toxin-that is, most $\mathrm{Kv}$ channels are blocked by the tethered $\alpha$ DTX in the dark state (Fig. 1d, compare cyan (blocked channel) and orange (unblocked channel) lines). Illumination leads to a rapid increase in unblocked ion channels, followed by a somewhat slower reblocking of those channels when illumination ceases. On the basis of our simulations we predict that a synthetic fusion protein, as we described above, will react to illumination with reasonably fast response times (s) and will allow fully reversible repeated activation/deactivation cycles.

Lumitoxins mediate optical modulation of cellular $\mathrm{K}^{+}$current. We synthesized a gene coding for a lumitoxin that contained Dendroaspis angusticeps $\alpha \mathrm{DTX}$, which specifically binds to Kv1.1 and Kv1.2 channels, connected to the A. sativa LOV2-J $\alpha$ domain (AsLOV2) via a 26-residue flexible linker. This fusion protein was targeted for the secretory pathway using a cleavable signal peptide and was anchored to the extracellular side of the cell membrane by a single-pass transmembrane domain derived from the human platelet-derived growth factor receptor (PDGF-R). We expressed aDTX-lumitoxins in cultured PC12 cells co-transfected with Kv1.2, and saw healthy expression (Fig. 2a), as might be expected, given that both AsLOV2-containing proteins and peptide toxins had previously been shown separately to express in mammalian cells. Whole-cell patch clamp recordings showed characteristic baseline voltage-dependent $\mathrm{K}^{+}$currents in a cell expressing $\alpha \mathrm{DTX}$-lumitoxins (Fig. 2b, left panel). Illumination of the same cell with modest levels $\left(500 \mu \mathrm{W} \mathrm{mm}^{-2}\right)$ of blue $(455 \mathrm{~nm})$ light increased the wholecell $\mathrm{K}^{+}$current approximately twofold within seconds (Fig. 2c, orange circles and Fig. 2b, middle panel). After cessation of illumination, the whole-cell $\mathrm{K}^{+}$current recovered to pre-illumination levels within $2 \mathrm{~min}$ (Fig. 2b, right panel).

As predicted by our model (Fig. 1), the majority of ion channels were blocked in the dark state, as judged by the baseline 


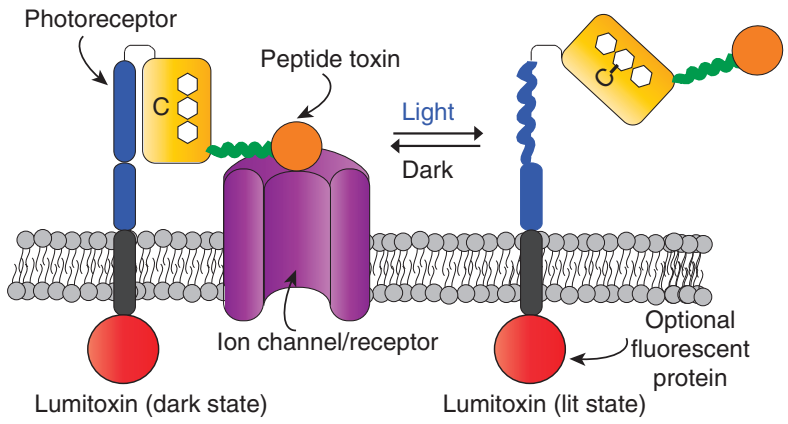

C

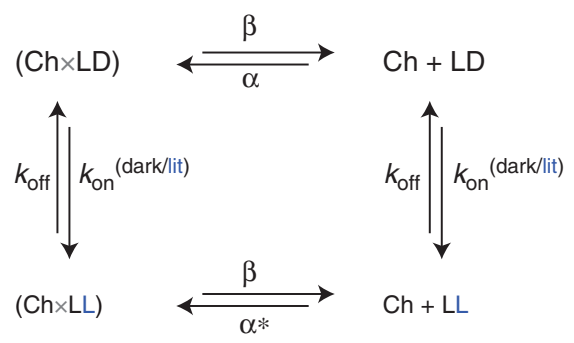

b
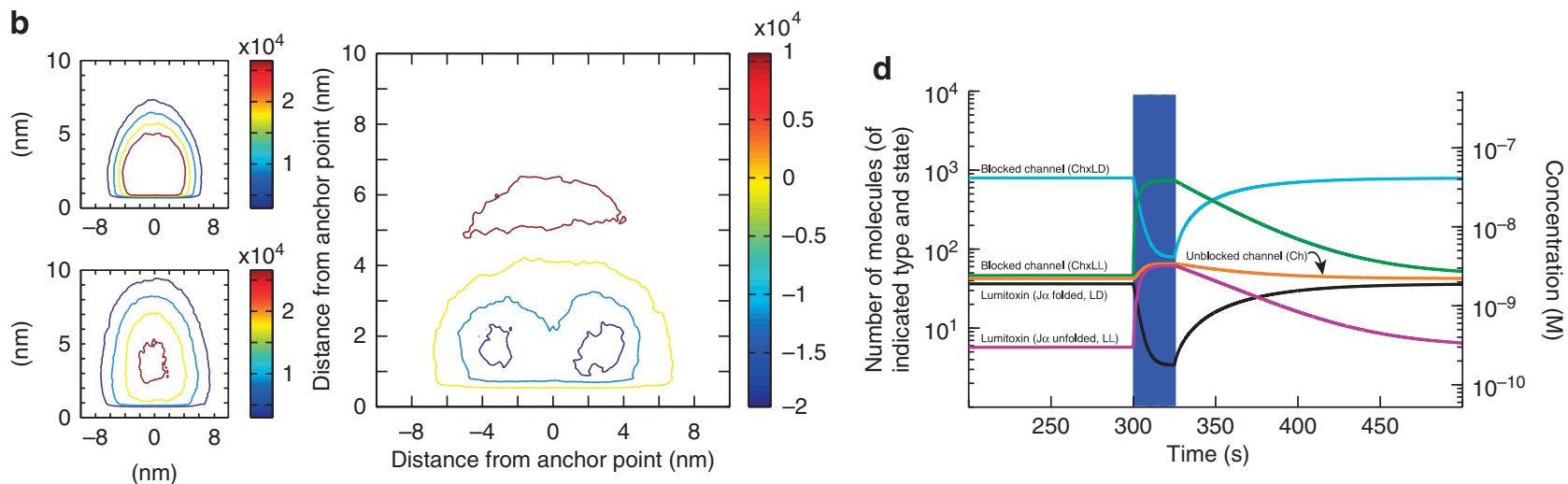

Figure 1 | A theoretical framework for light-actuated tethered protein ligands. (a) Architecture of fusion protein containing a membrane-anchored (PDGF-R, black) photoreceptor AsLOV2 (yellow; 'C' indicates the location of key cysteine, and molecular structure schematically represents flavin)/J $\alpha$ (blue) connected to a peptide toxin (orange) via a flexible linker (green wiggly line), optionally equipped with a fluorophore (red sphere).

(b) Tethered-particle motion model: location probability of encountering the peptide domain projected on the $x-z$ plane (that is, where the membrane is the $x-y$ plane) for a short (6/26, upper left panel) versus longer membrane tether ( $27 / 26$, lower left panel). Numbers before and after the ' $/$ ' indicate length, in amino acids, of the blue and green linkers, respectively. The difference (right panel) indicates that increasing the tether length decreases the peptide concentration close to the cell surface. (c) A model describing the binding equilibrium of a hypothetical protein adopting two different states (dark and lit) differing in biological activity (high affinity and low affinity) $-L D$ and LL, respectively, to an ion channel (Ch). (d) Simulation of the dynamic response of the model depicted in (c) to illumination, indicated by blue bar (see legends). See methods for model parameters.

$\mathrm{K}^{+}$currents recorded in cells co-expressing both $\alpha \mathrm{DTX}-$ lumitoxin and Kv1.2 versus cells expressing Kv1.2 alone (mean current at $+50 \mathrm{mV}: 40 \pm 12 \mathrm{pA} / \mathrm{pF}$ versus $206 \pm 24 \mathrm{pA} / \mathrm{pF}$, $P<0.0001$ two-tailed Student's $t$-test, $n=7-20)$. Furthermore, as predicted by our model, the whole-cell $\mathrm{K}^{+}$current rose within seconds and then, post illumination, attenuated back to baseline somewhat more slowly, but completely (Fig. 2c, orange circles).

An important aspect of the utility of peptide toxins in physiology and neuroscience is their excellent specificity; they can differentiate between subfamilies of closely related ion channels and receptors. To test whether the observed lightdependent current increase was ion channel-specific, we coexpressed the Shaker channel and $\alpha$ DTX-lumitoxin in the same cell. Kv1.2 and Shaker are similar in many aspects, but differ in their sensitivity to $\alpha$ DTX; $\alpha$ DTX binds to Kv1.2 with picomolar affinity, while its affinity for Shaker is very low (micromolar) ${ }^{25}$. As expected, illumination did not alter whole-cell $\mathrm{K}^{+}$current in cells that co-expressed Shaker and $\alpha$ DTX-lumitoxin, demonstrating that peptide toxins embedded within lumitoxins maintain their binding specificity (Fig. 2c, black circles). Neither were the properties of the AsLOV2 domain overtly perturbed by embedding within a lumitoxin, as current modulation was dependent on blue light, and was not affected by green light (Fig. 2d). We measured the time constant for light-driven lumitoxin effect-that is, the time constant constant by which the $\mathrm{J} \alpha$ helix unfolds and presumably lowers the local $\alpha \mathrm{DTX}$ concentration near the cell surface-leading to an increase in available Kv1.2 channels. We found the time constant to be a function of irradiance, consistent with $J \alpha$ helix unfolding being dependent on photon absorption. The apparent half-maximum time constant is achieved at $80 \mu \mathrm{W} \mathrm{mm}{ }^{-2}$ (Fig. 2e, left panel). Thus, note that $\mathrm{K}^{+}$current could be modulated by nondamaging, relatively low light intensities. On the other hand, the post-illumination recovery of $\mathrm{K}^{+}$current to pre-illumination baseline, associated with an increase in $\alpha \mathrm{DTX}$ concentration close to the membrane-bound ion channel and increased block of Kv1.2, solely depends on the spontaneous dark-state refolding of LOV2-J $\alpha$ and is thus expected to be independent of irradiance, which we confirmed experimentally (Fig. 2e, right panel). Repeated stimulation with blue light demonstrates that $\alpha$ DTXlumitoxins are able to carry out consecutive $\mathrm{K}^{+}$current modulations and are not undergoing destructive conformational changes (Fig. 2f).

The lumitoxin architecture enables rational protein design. We assessed the tunability of the lumitoxin architecture by utilizing a family of mutated $\alpha \mathrm{DTX}$ variants, which are known to possess altered affinity towards Kv1.2. High-affinity binding of $\alpha$ DTX depends crucially on the peptide residues K5 and L9, and changing them to alanine decreases the binding affinity by three orders of magnitude ${ }^{24}$. We thus expect two things when introducing the 
a

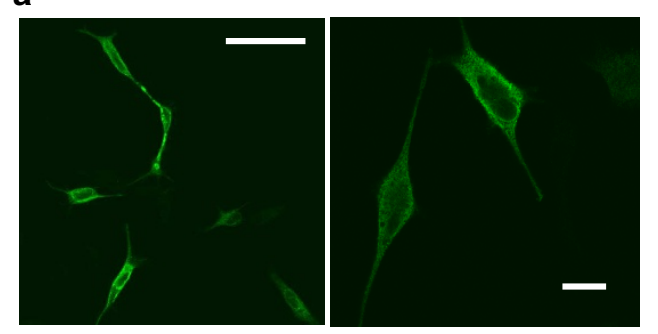

b

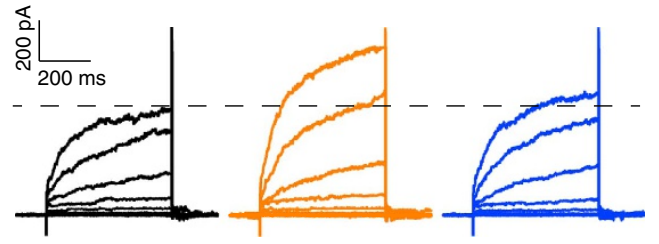

e

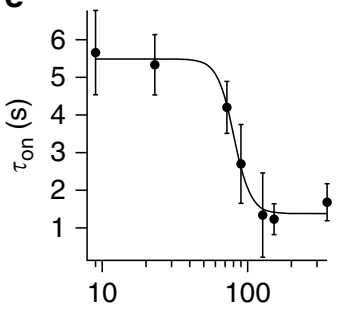

Irradiance $\left(\mu \mathrm{W} \mathrm{mm}{ }^{-2}\right)$

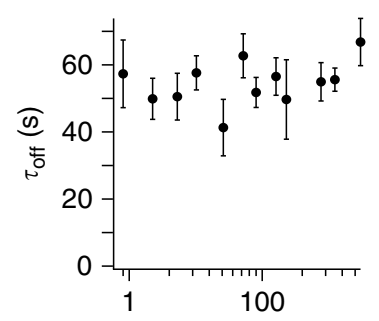

Irradiance $\left(\mu \mathrm{W} \mathrm{mm} \mathrm{mm}^{-2}\right.$
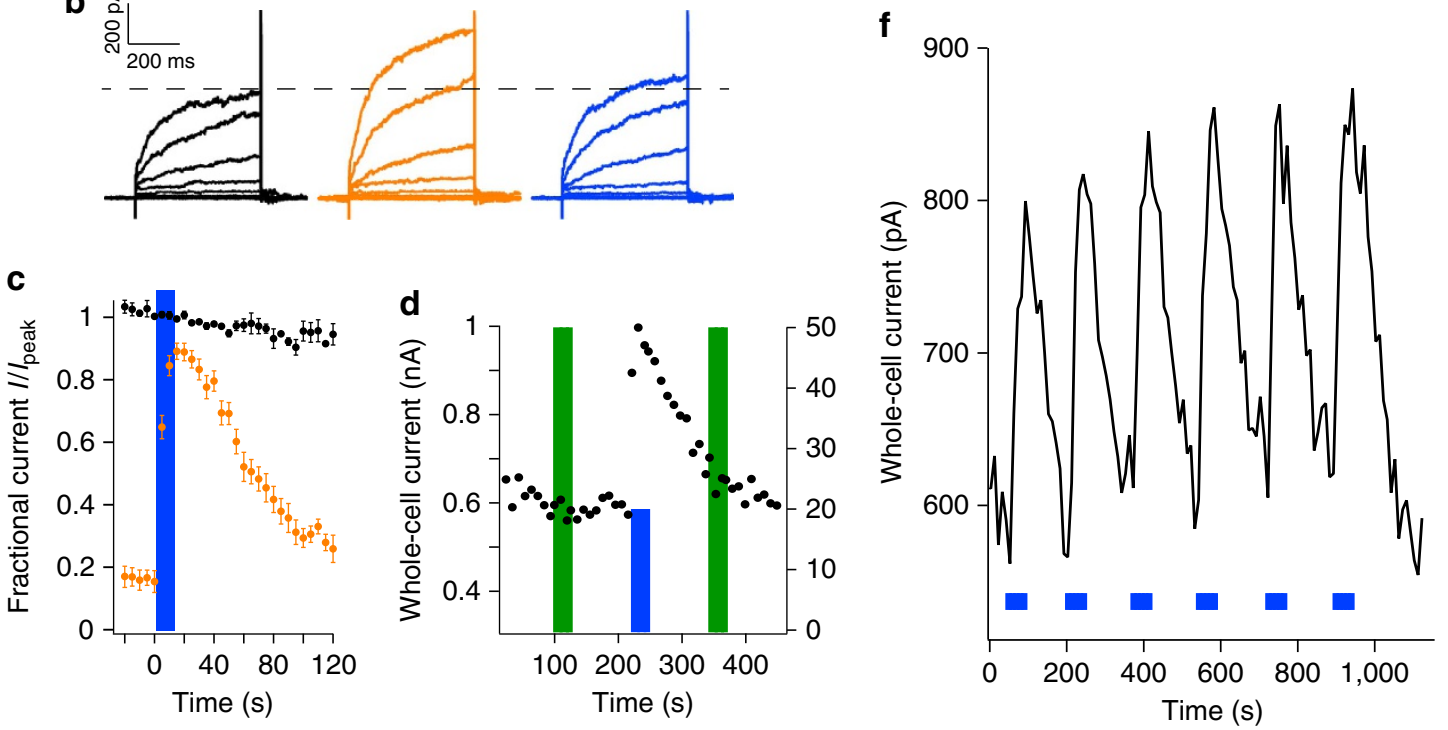

Figure 2 | Lumitoxins mediate light actuation of specific Kv channels. (a) PC12 cells expressing FLAG-tagged lumitoxins containing AsLOV2 and $\alpha$-Dendrotoxin $(\alpha \mathrm{DTX})$, here denoted dendrolumitoxin. Cells are fixed and stained with $\alpha$-FLAG/Alexa 488. Scale bar, $100 \mu \mathrm{m}$ (left panel), $20 \mu \mathrm{m}$ (right panel). (b) Whole-cell $\mathrm{K}^{+}$currents before (black), during (orange) and after (blue) illumination with $500 \mu \mathrm{W} \mathrm{mm}^{-2}$ blue (455 nm) light (colour and power used throughout, unless otherwise indicated). Holding voltage $-80 \mathrm{mV}$, depolarization voltages increasing in increments of $10 \mathrm{mV}$ to $+50 \mathrm{mV}$. (c) Normalized whole-cell $\mathrm{K}^{+}$current modulation (that is, current divided by maximum observed current $I_{\text {peak }}$ ) in response to blue-light illumination (blue bar) recorded in PC12 cells co-expressing dendrolumitoxin with Kv1.2 (orange) or Shaker (black). Plotted throughout is the mean plus or minus s.e.m., $n=3$ cells. (d) Dendrolumitoxin modulation of Kv1.2 responds to blue ( $455 \mathrm{~nm}$, blue bar) but not green ( $530 \mathrm{~nm}$, green bars) light. (e) Dependence of apparent $\tau_{\text {on }}$ and apparent $\tau_{\text {off }}$ of whole-cell Kv1.2 current modulation (that is, curves plotted as in $\mathbf{c}$ ) on irradiance. $n=2-4$ cells each point. (f) Repeated modulation of whole-cell Kv1.2 current with illumination (blue bars).

corresponding mutations K5A and L9A into $\alpha$ DTX-lumitoxins. First, we expect the initial $\mathrm{K}^{+}$current to increase, as our model predicts that decreasing the peptide affinity results in fewer channels being blocked in the dark (off) state. Second, we expect the activation ratio $\left(I_{\text {lit }} / I_{\text {dark }}\right)$ to decrease as fewer channels are now primed to become unmasked by illumination. We experimentally confirmed these expectations, and, after investigation of a suite of mutated $\alpha$ DTX-lumitoxins (Fig. 3a), we found that both baseline current and activation ratio depended monotonically on peptide toxin affinity, as would be expected given previous reports on free, that is non-tethered, toxin $^{24}$, and consistent with our predictions. For example, the mutations $\mathrm{R} 3 \mathrm{~A}$ and $\mathrm{R} 4 \mathrm{~A}$, which decrease the binding affinity 10 -fold for free $\alpha$ DTX, decreased the activation ratio by $20 \%$. The mutations $\mathrm{K} 5 \mathrm{~A}$ and $\mathrm{L} 9 \mathrm{~A}$, which decrease the binding affinity $>1,000$-fold, completely abolished the light-dependent increase in $\mathrm{K}^{+}$current (Fig. 3b).

We do not know whether mutations in $\alpha$ DTX affect the lumitoxin affinity through altering peptide toxin/channel association or dissociation. However, by using the model developed above, we can simulate the theoretical activation ratios of $\alpha$ DTXlumitoxin variants using either assumption and found that the effects of all mutations except $\mathrm{H} 10 \mathrm{~A}$ on the experimentally determined activation ratios were consistent with a decrease in the association rate constant (Fig. 3b, solid lines).

Lumitoxins are modular and channel specificity is adjustable. In the ideal case, it would be possible to tether multiple lightswitchable protein ligands to the cell membrane without any optimization of the non-ligand protein parts. Consequently, we explored whether the lumitoxin architecture fulfills this requirement by creating lumitoxin genes containing different peptide toxins that target distinct $\mathrm{Kv}$ channel types. Whereas $\alpha \mathrm{DTX}$ blocks both Kv1.1 and Kv1.2 channels ${ }^{28}$, Dendroaspis polylepis DTX-K, which shares only $63 \%$ sequence identity with $\alpha$ DTX, blocks mainly Kv1.1 channels ${ }^{29}$. Conkunitzin-S1 (CONK1), isolated from the marine cone snail Conus striatus, specifically blocks the Drosophila Shaker Kv channel ${ }^{30}$. We expressed the resulting lumitoxins in mammalian cell culture co-transfected with the Kv channels Kv1.1, Kv1.2 and Shaker. We used a variant of the Shaker $\mathrm{Kv}$ containing a mutation (K427D) shown to increase CONK1 affinity ${ }^{30}$. Whole-cell patch clamp electrophysiology reveals a clear specificity in the ability of lumitoxins to modulate $\mathrm{K}^{+}$currents in response to blue-light illumination $\left(500 \mu \mathrm{W} \mathrm{mm}{ }^{-2}\right)$. Whereas $\alpha$ DTX-containing 

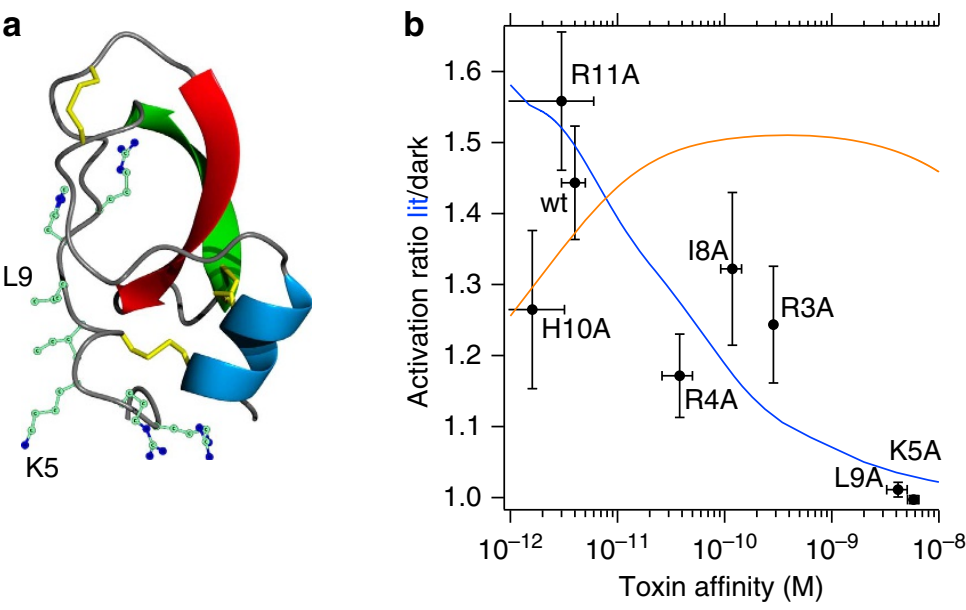

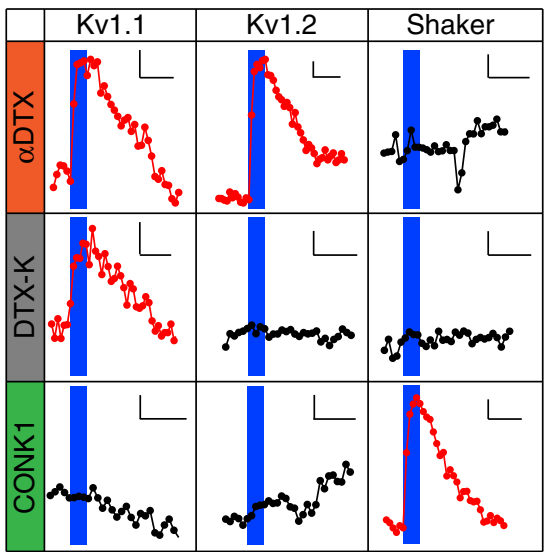

d

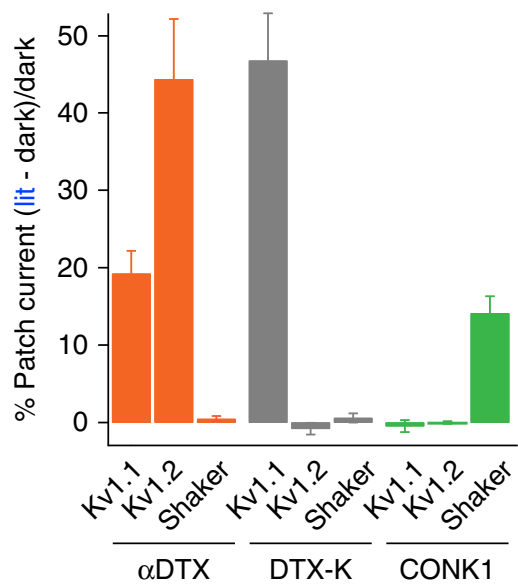

Figure 3 | Lumitoxin variants created through rational protein design can be incorporated into the modular lumitoxin architecture. (a) Mutated residues (shown as stick diagrams) mapped on the $\alpha$ DTX crystal structure (PDB accession number: 1DTX, shown as a cartoon). Residues labelled (K5, L9) are discussed in the text. (b) Average ratio of whole-cell $\mathrm{K}^{+}$current (Kv1.2 in PC12) during versus before illumination with $500 \mu \mathrm{Wmm}{ }^{-2}$ for $\alpha \mathrm{DTX}$ variants as a function of reported toxin affinity ${ }^{24}$. Plotted throughout is the mean plus or minus s.e.m., $n=7-20$ cells each point. Solid lines are simulated ratios assuming that mutations affect either solely the peptide toxin's association rate constant $\alpha$ (blue line) or the dissociation rate constant $\beta$ (orange line). (c) Representative current trace for indicated lumitoxin/channel combinations in response to illumination (blue bar). Inset scale bars: $50 \mathrm{pA}$ (ordinate), $50 \mathrm{~s}$ (abscissa). (d) Lumitoxin-specific increase in whole-cell patch in response to illumination with blue light ( $500 \mu \mathrm{W} \mathrm{mm}^{-2}$ ) for specific Kv channels. $n=3-20$.

lumitoxins affect both Kv1.1 and Kv1.2, but not Shaker (K427D) (Fig. 3c upper row, Fig. 3d), DTX-K-containing lumitoxins are specific for Kv1.1 (Fig. 3c middle row, Fig. 3d). Similarly, CONK1-containing lumitoxins only affect Shaker Kv channels, but not the mammalian homologs Kv1.1 or Kv1.2 (Fig. 3c bottom row, Fig. 3d). These results imply that lumitoxin specificity can be altered by swapping the genetically encoded protein ligand without the necessity of optimizing non-ligand protein domains, resulting in multiple genetically encoded reagents for the actuation of specific subclasses of endogenous Kv channels.

\section{Discussion}

We here present the architecture of a fully genetically encoded tool for modulating the concentration of a protein ligand near the surface of a specified cell or set of cells by light. The resultant 'lumitoxins' are fusion proteins of two fundamentally useful functional elements, peptide neurotoxins and the photoreceptor LOV2-J $\alpha$, which serves as the tether between the protein ligand and the membrane. We demonstrated the functionality of our technology by creating several genetically encoded peptide toxins, targeting different classes of $\mathrm{Kv}$ channels, whose concentration could be modulated by light, in a fully reversible manner. Our architecture was capable of yielding lumitoxins that modulated $\mathrm{K}^{+}$ currents with subfamily precision (for example, favouring Kv1.1 over Kv1.2 with perfect discrimination), demonstrating that peptide toxins, when part of a light-activated membrane-tether fusion protein, retain their activity and specificity. This specificity might be further facilitated by the addition of subcellular protein-trafficking motifs so that, for example, just the subset of Kv channels located on the axons or dendrites might be modulated. Exploring whether other classes of ion channels, and other membrane proteins such as neurotransmitter receptors, GPCRs, receptor tyrosine kinases and so forth, can be actuated by lumitoxins may represent a natural field of exploration in the future.

In earlier studies, the LOV domain had been customized in several ways to embed various different peptides and enzymes within or next to the LOV domain ${ }^{31-33}$. Our new architecture is modular in the sense that the LOV domain does not require customization for each target peptide and thus might present a more general solution to make peptides or proteins available 
Table 1 | Lumitoxin construct generation.

\section{Fusion protein component}

Secretion signal/FLAG tag

$\alpha \operatorname{DTX}$ (wt)

DTX-K

CONK1

Linker

LOV2-J $\alpha$

PDGF-R-mCherry

upon illumination. Another set of earlier studies fused protein ligands to membrane anchors, enabling fully genetically encoded blockade of endogenous ion channels ${ }^{9-11}$. Our architecture builds on these pioneering studies by enabling ion channel activity to be dynamically controlled over rapid timescales. Finally, a third line of past inquiry utilizes light-responsive, externally added
Amino acid sequence

MSALL ILALV GAAVA

DYKDD DDKL

QPRRK LCILH RNPGR CYDKI PAFYY NOKKK QCERF DWSGC GGNSN RFKTI EECRR TCIG RKIPS FYYKW KAKQC LPFDY SGCGG NANRF KTIEE CRRTC VG

KDRPS LCDLP ADSGS GTKAE KRIYY NSARK QCLRF DYTGQ GGNEN NFRRT YDCQR TCLYT

GTAAA DYKDD DDKID AAAGG ALCNE F

LATTL ERIEK NFVIT DPRLP DNPII FASDS FLQLT EYSRE EILGR NCRFL QGPET DRATV RKIRD AIDNQ TEVTV QLINY TKSGK KFWNL FHLQP MRDQK GDVQY FIGVQ LDGTE HVRDA AEREG VMLIK KTAEN IDEA AKEL

RVAVG QDTQE VIVVP HSLPF KVVVI SAILA LVVLT IISLI ILIML WQKKP RRIRM VSKGE EDNMA IIKEF MRFKV HMEGS VNGHE FEIEG EGEGR PYEGT QTAKL KVTKG GPLPF AWDIL SPQFM YGSKA YVKHP ADIPD YLKLS FPEGF KWERV MNFED GGVVT VTQDS SLQDG EFIYK VKLRG TNFPS DGPVM QKKTM GWEAS SERMY PEDGA LKGEIK QRLKL KDGGH YDAEV KTTYK AKKPV QLPGA YNVNI KLDIT SHNED YTIVE QYERA EGRHS TGGMD ELYK*
AAKYC KLPLR IGPCK

\section{DNA sequence}

atg agc gcc ctg ctg atc ctg gcc ctg gtg ggc gcc gcc gtg gec gac tac aag gac gac gac gac aag ctg cag $\mathrm{CCC}$ aga aga aag ctg tgc atc ctg cac aga aac ccc ggc aga tgc tac gac aag atc ccc gcc ttc tac tac aac cag aag aag aag cag tgc gag aga ttc gac tgg agc ggc tgc ggc ggc aac agc aac aga ttc aag acc atc gag gag tgc aga aga acc tgc atc ggc

gcc gcc aag tac tgc aag ctg ccc ctg aga atc ggt cca tgc aag cgg aag atc ccc agc ttc tac tac aag tgg aag gcc aag cag tgc ctg ccc ttc gac tac tct ggc tgc gga ggc aac gcc aac cgg ttc aag acc atc gag gaa tgc aga cgg acc tgc gtg gga

aag gac cgg $\mathrm{ccc}$ agc $\mathrm{ctg}$ tgc gac ctg cct gec gat tct ggc tct ggc acc aag gec gag aag cgg atc tac tac aac agc gcc cgg aag cag tgc ctg aga ttc gac tac aca ggc cag ggc ggc aac gag aac aac ttc cgg cgg acc tac gac tgc cag aga acc tgc ctg tac acc ggt acc gcc gcc gec gac tac aag gac gac gac gac aag atc gac gcc gcc gcc ggc ggc gcc ctg tgc aac ttg gct act aca ctt gaa cgt att gag aag aac ttt gtc att act gac cca aga ttg cca gat aat ccc att ata ttc gcg tcc gat agt ttc ttg cag ttg aca gaa tat agc cgt gaa gaa att ttg gga aga aac tgc agg ttt cta caa ggt cct gaa act gat cgc gcg aca gtg aga aaa att aga gat gcc ata gat aac caa aca gag gtc act gtt cag ctg att aat tat aca aag agt ggt aaa aag ttc tgg aac ctc ttt cac ttg cag cct atg cga gat cag aag gga gat gtc cag tac ttt att ggg gtt cag ttg gat gga act gag cat gtc cga gat gct gcc gag aga gag gga gtc atg ctg att aag aaa act gca gaa aat att gat gag gcg gca aaa gaa ctt

cga gtt gct gtg ggc cag gac acg cag gag gtc atc gtg gtg cca cac tcc ttg ccc ttt aag gtg gtg gtg atc tca gcc atc ctg gcc ctg gtg gtg ctc acc atc atc tcc ctt atc atc ctc atc atg ctt tgg cag aag aaa cca cgt agg att cgt atg gtg agc aag ggc gag gag gat aac atg gcc atc atc aag gag ttc atg cgc ttc aag gtg cac atg gag ggc tcc gtg aac ggc cac gag ttc gag atc gag gge gag ggc gag ggc cgc ccc tac gag ggc acc cag acc gcc aag ctg aag gtg acc aag ggt ggc CCC ctg CCC ttc gcc tgg gac atc ctg tcc cct cag ttc atg tac ggc tcc aag gcc tac gtg aag cac CCC gCc gac atc CCC gac tac ttg aag ctg tcc ttc ccc gag ggc ttc aag tgg gag cgc gtg atg aac ttc gag gac ggc gge gtg gtg acc gtg acc cag gac tcc tcc ctg cag gac ggc gag ttc atc tac aag gtg aag ctg cgc ggc acc aac ttc ccc tcc gac ggc CCC gta atg cag aag aag acc atg ggc tgg gag gcc tcc tcc gag cgg atg tac CCC gag gac ggc gec ctg aag ggc gag atc aag cag agg ctg aag ctg aag gac ggc ggc cac tac gac get gag gtc aag acc acc tac aag gcc aag aag ccc gtg cag ctg ccc ggc gcc tac aac gtc aac atc aag ttg gac atc acc tcc cac aac gag gac tac acc atc gtg gaa cag tac gaa cgc gcc gag ggc cgc cac tcc acc ggc ggc atg gac gag ctg tac aag taa

CONK1, Conkunitzin-S1; DTX, $\alpha$-Dendrotoxin; LOV2, light-oxygen-voltage domain; PDGF-R, platelet-derived growth factor receptor.

chemical ligands anchored to specific sites engineered into ion channels ${ }^{13-19}$, enabling fast induction and reversal, by modulating the concentration of the ligand relative to the ion channel surface. Our current methodology extends these ideas by being fully genetically encoded, eliminating the need for exogenous chemical application. 
We developed an analytical model for lumitoxin function, based on biophysical principles, and focusing on the hypothesis that the illumination of the lumitoxin would, as the LOV2-J $\alpha$ unfolds, modulate the local concentration of the peptide toxin near the cell surface when expressed on cellular membranes. In our theory, the signal magnitude is not determined by the detailed interactions between the LOV2 domain and the coupled effector domain (here, a peptide toxin), as in past LOV-based optogenetic tools ${ }^{31-34}$, but rather by the difference in length and rotational freedom of the J $\alpha$ helix between lit and dark states, which directly influences the difference in local concentration near the cell surface. Both the apparent forward and backward rates of the $\alpha$ DTX-containing lumitoxin are similar to the unfolding and folding kinetics of the isolated AsLOV2 domain ${ }^{26,27}$, hinting at the rate-limiting step in light switching for lumitoxins utilizing AsLOV2 as the photoswitch. Our models, protein ligand swaps, and mutagenesis data suggest that the lumitoxin architecture is sufficiently modular for AsLOV2 properties such as kinetics, helix folding equilibrium, and so on to be engineered independent of protein ligand and anchor context, allowing for upgrades or altered performance of lumitoxins. Thus, our strategy represents a potentially important modular and tunable architecture in the field of engineered light-activated proteins. Note that, in this context, the high light sensitivity of lumitoxins (activated by as little as $10 \mu \mathrm{W} \mathrm{mm}{ }^{-2}$ ) relative to 'traditional' optogenetic tools such as ChR2 (routinely driven by $>1,000 \mu \mathrm{W} \mathrm{mm}{ }^{-2}$ ) may enable lumitoxins to be incorporated into existing experimental contexts without requiring disturbance of other engineered signaling pathways.

Systematic studies of a variety of different protein ligands, together with other structural features of lumitoxins such as linker length, composition and choice of membrane anchor, are needed to expand the family of lumitoxin reagents and improve its functional characteristic. Lumitoxins capable of blockading channels in response to light, in addition to mediating their actuation, would also be particularly useful. In the future, the lumitoxin architecture, or future variants thereof, might be utilized to tether arbitrary genetically encoded payloads beyond $\mathrm{Kv}$ channel modulating protein ligands-neuropeptides, growth factors, signaling domains-close to membrane-associated ion channels and receptors without requiring extensive re-optimization of the protein architecture.

\section{Methods}

Modelling tethered-particle motion and lumitoxin stimulation. A system of two spheres, representing a LOV2 domain ( $2 \mathrm{~nm}$ diameter) and a peptide toxin ( $0.75 \mathrm{~nm}$ diameter), connected to each other and a solid wall via linkers with a variable number of elements $(0.2 \mathrm{~nm}$ diameter) representing linker amino acids, was simulated in MATLAB. Each linker element $(n)$ was connected to its neigh bouring linker elements $(n+1$ and $n-1)$ with springs (spring constant $\left.0.5 \mathrm{~N} \mathrm{~m}^{-1}\right)$ at the distance of a peptide bond $(0.4 \mathrm{~nm})$. Pairs of second nearest neighbour elements were also connected to each other with a spring (spring constant $0.25 \mathrm{~N} \mathrm{~m}^{-1}$ ) in order to maintain the equilibrium bond angle between them at 109 degrees. Fluctuations of this system because of Brownian motion took the form $v \cdot \frac{\mathrm{d} r}{\mathrm{~d} t}=F_{\text {separation }}+F_{\text {bond angle }}+F_{\text {overlap }}+F_{\text {random }}$ and were simulated for $150 \mathrm{~ns}$ for several combinations of lengths of linkers.

Modelling of lumitoxin stimulation was performed using MATLAB scripts numerically integrating a set of ordinary differential equations describing the thermodynamic model depicted in Fig. 1c. Simulation parameters were $n($ Toxin $)=n($ Channel $)=8,000$; reaction volume $=3.2 \times 10^{-14} 1$ (corresponding to a $13-\mu \mathrm{m}$ (inner radius) hollow sphere with $15 \mathrm{~nm}$ wall thickness); $\alpha=2 \times 10^{8}$ $\mathrm{M}^{-1} \mathrm{~s}^{-1} ; \alpha^{*}=6.6 \times 10^{7} \mathrm{M}^{-1} \mathrm{~s}^{-1} ; \beta=2 \times 10^{-2} \mathrm{~s}^{-1} ; k_{\text {on }}($ lit $)=2.5 \times 10^{-1} \mathrm{~s}^{-1}$, $k_{\text {on }}($ dark $)=1.6 \times 10^{-3} \mathrm{~s}^{-1}, k_{\text {off }}=2.55 \times 10^{-2} \mathrm{~s}^{-1}$. For theoretical activation ratios (Fig. 3b) $\alpha$ ranged from $5 \times 10^{4}$ to $4 \times 10^{8} \mathrm{M}^{-1} \mathrm{~s}^{-1}, \alpha^{*}$ ranged from $1.7 \times 10^{4}$ to $1.4 \times 10^{8} \mathrm{M}^{-1} \mathrm{~s}^{-1}$, and $\beta$ ranged from $2 \times 10^{-4}$ to $4 \mathrm{~s}^{-1}$.

Molecular biology and construct generation. Genes encoding for fusion proteins were assembled as a multicomponent cloning cassette from annealed oligonucleotides (IDT DNA) containing these elements (in order): BglII or NheI-Secretion Signal/FLAG tag-HindIII-( $\alpha$ DTX or DTX-K or CONK1)-KpnI-Linker-
LOV2-J $\alpha(404-546)$-NotI-PDGF-R-mCherry-XbaI. PDGF-R-mCherry was derived from pFU-MVIIA-PC (Addgene) ${ }^{10}$. See Table 1 for sequence details. This cassette was inserted into the mammalian expression vector pcDNA3.1 $(+)$ (Invitrogen) using NheI/XbaI restriction sites. The respective genes coding for rat Kv1.2 (Kv1.2), rat Kv1.1 (Kv1.1) or Shaker were amplified from Kv1.2-pBluescript, Shaker-pBluescript (gifts from Roderick Mackinnon), or BacMam Kv1.1 (Invitrogen) and inserted into pcDNA3.1 $(+)$ or pEGFP-N3 using BamHI/EcoRI or NheI/EcoRI, respectively. Both $\alpha \mathrm{DTX}$-lumitoxin and Kv1.2 cassettes were also inserted into the bidirectional expression vector pBI-CMV1 (Clontech) using BglII/ $\mathrm{Xba \textrm {I }}$ and $\mathrm{BamHI} /$ NotI sites, respectively, to drive expression from the same plasmid.

Mammalian cell culture. PC12 cells (ATCC) were maintained in DMEM (Cellgro), 10\% fetal bovine serum (Invitrogen), 5\% donor horse serum (Invitrogen), 1\% penicillin/streptomycin (Cellgro) and 1\% sodium pyruvate (Biowhittaker). For electrophysiological recordings and imaging, cells were plated on glass coverslips treated with Matrigel (BD Bioscience). Adherent cells were transfected using Lipofectamine LTX (Invitrogen) following the manufacturer's instructions and recorded $36-48 \mathrm{~h}$ later.

For confocal microscopy, cells were fixed with $4 \%$ formaldehyde, permeabilized with $0.4 \%$ saponin and either stained with anti-FLAG M2 peptide antibody (Sigma A2220, 1:1,000 dilution) followed by anti-mouse-Alexa488 secondary antibody staining (Invitrogen) or directly observed using mCherry fluorescence.

Electrophysiology and illumination. $\mathrm{K}^{+}$currents were recorded from PC12 cells 36-48 post-transfection using whole-cell voltage clamp. Analog signals were filtered $(1-5 \mathrm{kHz})$ using the built-in 4-pole Bessel filter of an Axopatch 200B patch clamp amplifier (Molecular Devices), digitized at $10 \mathrm{kHz}$ (Digidata $1440 \mathrm{~A}$, Molecular Devices) and stored on a computer hard disk. The bath solution contained the following (mM): $125 \mathrm{NaCl}, 2 \mathrm{KCl}, 3 \mathrm{CaCl}_{2}, 1 \mathrm{MgCl}_{2}, 10 \mathrm{HEPES}$, 30 glucose, adjusted to $\mathrm{pH} 7.3$ with $\mathrm{NaOH}$. Electrodes were drawn from borosilicate patch glass (Warner Instruments) to a resistance of 5-10 $\mathrm{M} \Omega$. Patches with access resistance of $>50 \mathrm{M} \Omega$ were discarded from the data analysis The reported $n$ refers to the number of patched cells.

Cells were screened for mCherry expression using a 565-nm high-power LED (Thorlabs) filtered by a $560 \pm 40$-nm bandpass filter (Semrock) through a $40 \times$ lens. Lumitoxins were stimulated with a $455-\mathrm{nm}$ high-power LED (Thorlabs).

\section{References}

1. Kullmann, D. M. Neurological channelopathies. Annu. Rev. Neurosci. 33, 151-172 (2010).

2. Abbott, G. W. Molecular mechanisms of cardiac voltage-gated potassium channelopathies. Curr. Pharm. Des. 12, 3631-3644 (2006).

3. Kini, R. M. \& Doley, R. Structure, function and evolution of three-finger toxins: Mini proteins with multiple targets. Toxicon 56, 855-867 (2010).

4. Koh, D. C. I., Armugam, A. \& Jeyaseelan, K. Snake venom components and their applications in biomedicine. Cell. Mol. Life Sci. 63, 3030-3041 (2006).

5. Nirthanan, S. \& Gwee, M. C. E. Three-finger alpha-neurotoxins and the nicotinic acetylcholine receptor, forty years on. J. Pharmacol. Sci. 94, 1-17 (2004).

6. Terlau, H. \& Olivera, B. M. Conus venoms: a rich source of novel ion channeltargeted peptides. Physiol. Rev. 84, 41-68 (2004).

7. Corzo, G. \& Escoubas, P. Pharmacologically active spider peptide toxins. Cell. Mol. Life Sci. 60, 2409-2426 (2003).

8. Blumenthal, K. M. \& Seibert, A. L. Voltage-gated sodium channel toxins: poisons, probes, and future promise. Cell Biochem. Biophys. 38, 215-238 (2003).

9. Ibanez-Tallon, I. et al. Tethering naturally occurring peptide toxins for cell autonomous modulation of ion channels and receptors in vivo. Neuron 43, 305-311 (2004).

10. Auer, S. et al. Silencing neurotransmission with membrane-tethered toxins. Nat. Methods 7, 229-236 (2010).

11. Stürzebecher, A. S. et al. RAPID REPORT: An in vivo tethered toxin approach for the cell-autonomous inactivation of voltage-gated sodium channel currents in nociceptors. J. Physiol. (Lond) 588, 1695-1707 (2010).

12. Wu, Y., Cao, G., Pavlicek, B., Luo, X. \& Nitabach, M. N. Phase coupling of a circadian neuropeptide with rest/activity rhythms detected using a membranetethered spider toxin. PLoS Biol. 6, e273 (2008).

13. Banghart, M., Borges, K., Isacoff, E., Trauner, D. \& Kramer, R. H. Lightactivated ion channels for remote control of neuronal firing. Nat. Neurosci. 7, 1381-1386 (2004).

14. Volgraf, M. et al. Allosteric control of an ionotropic glutamate receptor with an optical switch. Nat. Chem. Biol. 2, 47-52 (2006).

15. Szobota, S. et al. Remote control of neuronal activity with a light-gated glutamate receptor. Neuron 54, 535-545 (2007).

16. Zemelman, B. V., Nesnas, N., Lee, G. A. \& Miesenböck, G. Photochemical gating of heterologous ion channels: remote control over genetically designated populations of neurons. Proc. Natl Acad. Sci. USA 100, 1352-1357 (2003). 
17. Lima, S. Q. \& Miesenböck, G. Remote control of behavior through genetically targeted photostimulation of neurons. Cell 121, 141-152 (2005).

18. Sandoz, G., Levitz, J., Kramer, R. H. \& Isacoff, E. Y. Optical control of endogenous proteins with a photoswitchable conditional subunit reveals a role for TREK1 in GABA(B) signaling. Neuron 74, 1005-1014 (2012).

19. Janovjak, H., Szobota, S., Wyart, C., Trauner, D. \& Isacoff, E. Y. A light-gated, potassium-selective glutamate receptor for the optical inhibition of neuronal firing. Nat. Neurosci. 13, 1027-1032 (2010).

20. Doyle, D. A. et al. The structure of the potassium channel: molecular basis of $\mathrm{K}+$ conduction and selectivity. Science 280, 69-77 (1998).

21. Long, S. B., Tao, X., Campbell, E. B. \& Mackinnon, R. Atomic structure of a voltage-dependent $\mathrm{K}+$ channel in a lipid membrane-like environment. Nature 450, 376-382 (2007).

22. Christie, J. M. Phototropin blue-light receptors. Annu. Rev. Plant Biol. 58, 21-45 (2007).

23. Möglich, A., Yang, X., Ayers, R. A. \& Moffat, K. Structure and function of plant photoreceptors. Annu. Rev. Plant Biol. 61, 21-47 (2010).

24. Gasparini, S. et al. Delineation of the functional site of alpha-dendrotoxin. The functional topographies of dendrotoxins are different but share a conserved core with those of other Kvl potassium channel-blocking toxins. J. Biol. Chem. 273, 25393-25403 (1998).

25. Tytgat, J., Debont, T., Carmeliet, E. \& Daenens, P. The alpha-dendrotoxin footprint on a mammalian potassium channel. J. Biol. Chem. 270, 24776-24781 (1995).

26. Salomon, M., Christie, J. M., Knieb, E., Lempert, U. \& Briggs, W. R. Photochemical and mutational analysis of the FMN-binding domains of the plant blue light receptor, phototropin. Biochemistry 39, 9401-9410 (2000).

27. Strickland, D. et al. Rationally improving LOV domain-based photoswitches Nat. Methods 7, 623-626 (2010).

28. Harvey, A. L. Twenty years of dendrotoxins. Toxicon 39, 15-26 (2001).

29. Robertson, B., Owen, D., Stow, J., Butler, C. \& Newland, C. Novel effects of dendrotoxin homologues on subtypes of mammalian Kv1 potassium channels expressed in Xenopus oocytes. FEBS Lett. 383, 26-30 (1996).

30. Bayrhuber, M. et al. Conkunitzin-S1 is the first member of a new Kunitz-type neurotoxin family. Structural and functional characterization. J. Biol. Chem. 280, 23766-23770 (2005).

31. Lungu, O. I. et al. Designing photoswitchable peptides using the AsLOV2 domain. Chem. Biol. 19, 507-517 (2012).
32. Wu, Y. I. et al. A genetically encoded photoactivatable Rac controls the motility of living cells. Nature 461, 104-108 (2009).

33. Renicke, C., Schuster, D., Usherenko, S. \& Essen, L. O. A LOV2 domain-based optogenetic tool to control protein degradation and cellular function. Chem. Biol. 20, 619-626 (2013).

34. Strickland, D., Moffat, K. \& Sosnick, T. R. Light-activated DNA binding in a designed allosteric protein. Proc. Natl Acad. Sci. USA 105, 10709-10714 (2008).

\section{Acknowledgements}

This work was supported by NIH Grants NIH 1DP2OD002002, NIH 1R01DA029639, NIH 1R01NS075421, NIH 1RC1MH088182, the NSF CAREER Award CBET 1053233 DARPA Living Foundries Contract HR0011-12-C-0068 and the New York Stem Cell Foundation-Robertson Investigator Award. D.S. was supported by the Damon Runyon Cancer Research Foundation (DRG 2095-11). P.W.T was supported by the Fannie and John Hertz Foundation. F.C. was supported by the National Science Foundation Graduate Research Fellowship under grant no. 1122374 and the Synthetic Intelligence Project.

\section{Author contributions}

D.S. and E.S.B. designed the experiments and wrote the manuscript. D.S. performed the experiments. F.C. and P.W.T. implemented the tethered particle simulation.

\section{Additional information}

Accession codes: Construct sequences have been deposited in the DDBJ/EMBL/ GenBank nucleotide database under accession codes KF878105 (aDTX-Lumitoxin), KF878106 (CONK1-Lumitoxin) and KF87810 (DTX-K-Lumitoxin) and are available on request at http://syntheticneurobiology.org/protocols.

Competing financial interests: The authors declare no competing financial interests.

Reprints and permission information is available online at http://npg.nature.com/ reprintsandpermissions/

How to cite this article: Schmidt, D et al. A fully genetically encoded protein architecture for optical control of peptide ligand concentration. Nat. Commun. 5:3019 doi: 10.1038/ncomms4019 (2014). 